\title{
Mean-Value Theorems for Harmonic Functions on the Cube in $\mathbb{R}^{n}$
}

\section{Petar Petrov}

Faculty of Mathematics and Informatics, Sofia University, Sofia, Bulgaria

Email: peynov@fmi.uni-sofia.bg

Received 4 August 2015; accepted 13 September 2015; published 16 September 2015

Copyright (C) 2015 by author and Scientific Research Publishing Inc.

This work is licensed under the Creative Commons Attribution International License (CC BY).

http://creativecommons.org/licenses/by/4.0/

(c) (i) Open Access

\section{Abstract}

Let $I_{n}(r)=\left\{x \in \mathbb{R}^{n}|| x_{i} \mid \leq r, i=1,2, \cdots, n\right\}$ be a hypercube in $\mathbb{R}^{n}$. We prove theorems concerning mean-values of harmonic and polyharmonic functions on $I_{n}(r)$, which can be considered as natural analogues of the famous Gauss surface and volume mean-value formulas for harmonic functions on the ball in $\mathbb{R}^{n}$ and their extensions for polyharmonic functions. We also discuss an application of these formulas-the problem of best canonical one-sided $L^{1}$-approximation by harmonic functions on $I_{n}(r)$.

\section{Keywords}

Harmonic Functions, Polyharmonic Functions, Hypercube, Quadrature Domain, Best One-Sided Approximation

\section{Introduction}

This note is devoted to formulas for calculation of integrals over the $n$-dimensional hypercube centered at $\mathbf{0}$

$$
I_{n}:=I_{n}(r):=\left\{\boldsymbol{x} \in \mathbb{R}^{n}|| x_{i} \mid \leq r, i=1,2, \cdots, n\right\}, r>0,
$$

and its boundary $P_{n}:=P_{n}(r):=\partial I_{n}(r)$, based on integration over hyperplanar subsets of $I_{n}$ and exact for harmonic or polyharmonic functions. They are presented in Section 2 and can be considered as natural analogues on $I_{n}$ of Gauss surface and volume mean-value formulas for harmonic functions ([1]) and Pizzetti formula [2], ([3], Part IV, Ch. 3, pp. 287-288) for polyharmonic functions on the ball in $\mathbb{R}^{n}$. Section 3 deals with the best one-sided $L^{1}$-approximation by harmonic functions. 
Let us remind that a real-valued function $f$ is said to be harmonic (polyharmonic of degree $m \geq 2$ ) in a given domain $\Omega \subset \mathbb{R}^{n}$ if $f \in C^{2}(\Omega)\left(f \in C^{2 m}(\Omega)\right)$ and $\Delta f=0 \quad\left(\Delta^{m} f=0\right)$ on $\Omega$, where $\Delta$ is the Laplace operator and $\Delta^{m}$ is its $m$-th iterate

$$
\Delta f:=\sum_{i=1}^{n} \frac{\partial^{2} f}{\partial x_{i}^{2}}, \quad \Delta^{m} f:=\Delta\left(\Delta^{m-1} f\right) .
$$

For any set $D \subset \mathbb{R}^{n}$, denote by $\mathcal{H}(D)\left(\mathcal{H}^{m}(D), m \geq 2\right)$ the linear space of all functions that are harmonic (polyharmonic of degree $m$ ) in a domain containing $D$. The notation $\mathrm{d} \lambda_{n}$ will stand for the Lebesgue measure in $\mathbb{R}^{n}$.

\section{Mean-Value Theorems}

Let $B_{n}(r):=\left\{\boldsymbol{x} \in \mathbb{R}^{n} \mid\|x\|:=\left(\sum_{i=1}^{n} x_{i}^{2}\right)^{1 / 2} \leq r\right\}$ and $S_{n}(r):=\left\{\boldsymbol{x} \in \mathbb{R}_{n} \mid\|x\|=r\right\}$ be the ball and the hypersphere in $\mathbb{R}^{n}$ with center $\mathbf{0}$ and radius $r$. The following famous formulas are basic tools in harmonic function theory and state that for any function $h$ which is harmonic on $B_{n}(r)$ both the average over $S_{n}(r)$ and the average over $B_{n}(r)$ are equal to $h(\mathbf{0})$.

The surface mean-value theorem. If $h \in \mathcal{H}\left(B_{n}(r)\right)$, then

$$
\frac{1}{\sigma_{n-1}\left(S_{n}(r)\right)} \int_{S_{n}(r)} h \mathrm{~d} \sigma_{n-1}=h(\mathbf{0}),
$$

where $\mathrm{d} \sigma_{n-1}$ is the $(n-1)$-dimensional surface measure on the hypersphere $S_{n}(r)$.

The volume mean-value theorem. If $h \in \mathcal{H}\left(B_{n}(r)\right)$, then

$$
\frac{1}{\lambda_{n}\left(B_{n}(r)\right)} \int_{B_{n}(r)} h \mathrm{~d} \lambda_{n}=h(\mathbf{0}) .
$$

The balls are known to be the only sets in $\mathbb{R}^{n}$ satisfying the surface or the volume mean-value theorem. This means that if $\Omega \subset \mathbb{R}^{n}$ is a nonvoid domain with a finite Lebesgue measure and if there exists a point $x_{0} \in \Omega$ such that $h\left(x_{0}\right)=\frac{1}{\lambda_{n}(\Omega)} \int_{\Omega} h \mathrm{~d} \lambda_{n}$ for every function $h$ which is harmonic and integrable on $\Omega$, then $\Omega$ is an open ball centered at $\boldsymbol{x}_{0}$ (see [4]). The mean-value properties can also be reformulated in terms of quadrature domains [5]. Recall that $\Omega \subset \mathbb{R}^{n}$ is said to be a quadrature domain for $\mathcal{H}(\Omega)$, if $\Omega$ is a connected open set and there is a Borel measure $\mathrm{d} \mu$ with a compact support $K_{\mu} \subset \Omega$ such that $\int_{\Omega} f \mathrm{~d} \lambda_{n}=\int_{K_{\mu}} f \mathrm{~d} \mu$ for every $\lambda_{n}$ integrable harmonic function $f$ on $\Omega$. Using the concept of quadrature domains, the volume mean-value property is equivalent to the statement that any open ball in $\mathbb{R}^{n}$ is a quadrature domain and the measure $\mathrm{d} \mu$ is the Dirac measure supported at its center. On the other hand, no domains having "corners" are quadrature domains [6]. From this point of view, the open hypercube $I_{n}^{\circ}$ is not a quadrature domain. Nevertheless, it is proved in Theorem 1 below that the closed hypercube $I_{n}$ is a quadrature set in an extended sense, that is, we find explicitly a measure $\mathrm{d} \mu$ with a compact support $K_{\mu}$ having the above property with $\Omega$ replaced by $I_{n}$ but the condition $K_{\mu} \subset I_{n}^{\circ}$ is violated exactly at the "corners" (for the existence of quadrature sets see [7]). This property of $I_{n}$ is of crucial importance for the best one-sided $L^{1}$-approximation with respect to $\mathcal{H}\left(I_{n}\right)$ (Section 3).

Let us denote by $D_{n}^{i j}$ the $(n-1)$-dimensional hyperplanar segments of $I_{n}$ defined by

$$
D_{n}^{i j}:=D_{n}^{i j}(r):=\left\{\boldsymbol{x} \in I_{n}|| x_{k}|\leq| x_{i}|=| x_{j} \mid, k \neq i, j\right\}, \quad 1 \leq i<j \leq n,
$$

(see Figure 1). Denote also

$$
\omega_{k}(\boldsymbol{x}):=\frac{\left(r-\max \left\{\left|x_{1}\right|,\left|x_{2}\right|, \cdots,\left|x_{n}\right|\right\}\right)^{k}}{k !}, \quad k \geq 0,
$$

and $\mathrm{d} \lambda_{m}^{\omega_{k}}:=\omega_{k} \mathrm{~d} \lambda_{m}$. It can be calculated that 


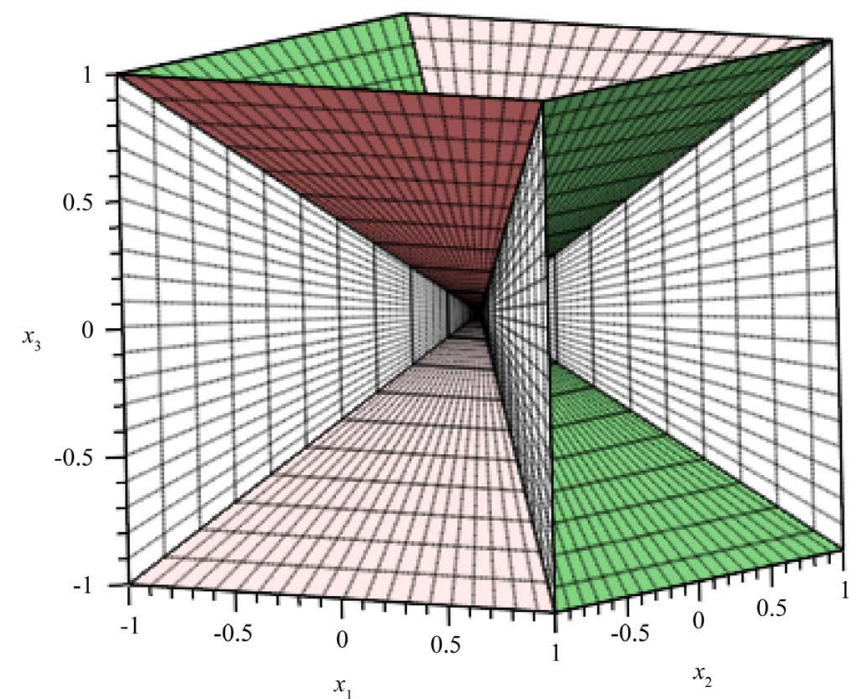

Figure 1. The sets $D_{3}^{12}(1)$ (white), $D_{3}^{13}(1)$ (green) and $D_{3}^{23}(1)$ (coral).

$$
\lambda_{n}^{\omega_{k}}\left(I_{n}\right)=2^{n} n ! \frac{r^{n+k}}{(n+k) !}, \quad \lambda_{n-1}^{\omega_{k}}\left(P_{n}\right)=2^{n} n ! \frac{r^{n+k-1}}{(n+k-1) !},
$$

and

$$
\lambda_{n-1}^{\omega_{k}}\left(D_{n}\right)=2^{n-1} n ! \frac{r^{n+k-1}}{(n+k-1) !}, \text { where } D_{n}:=\bigcup_{1 \leq i<j \leq n} D_{n}^{i j} .
$$

The following holds true.

Theorem 1 If $h \in \mathcal{H}\left(I_{n}\right)$, then $h$ satisfies:

(i) Surface mean-value formula for the hypercube

$$
\frac{1}{\lambda_{n-1}\left(P_{n}\right)} \int_{P_{n}} h \mathrm{~d} \lambda_{n-1}=\frac{1}{\lambda_{n-1}\left(D_{n}\right)} \int_{D_{n}} h \mathrm{~d} \lambda_{n-1},
$$

(ii) Volume mean-value formula for the hypercube

$$
\frac{1}{\lambda_{n}^{\omega_{k}}\left(I_{n}\right)} \int_{I_{n}} h \mathrm{~d} \lambda_{n}^{\omega_{k}}=\frac{1}{\lambda_{n-1}^{\omega_{k+1}}\left(D_{n}\right)} \int_{D_{n}} h \mathrm{~d} \lambda_{n-1}^{\omega_{k+1}}, \quad k \geq 0 .
$$

In particular, both surface and volume mean values of $h$ are attained on $D_{n}$.

Proof. Set

$$
M_{i}:=M_{i}(\boldsymbol{x}):=\max _{j \neq i}\left|x_{j}\right|,
$$

and

$$
\boldsymbol{x}_{t}^{i}:=\left(x_{1}, \cdots, x_{i-1}, t, x_{i+1}, \cdots, x_{n}\right) .
$$

Using the harmonicity of $h$, we get for $k \geq 1$

$$
\begin{aligned}
0 & =\int_{I_{n}} \Delta h \mathrm{~d} \lambda_{n}^{\omega_{k}}=\sum_{i=1}^{n} \int_{I_{n}} \omega_{k} \frac{\partial^{2} h}{\partial x_{i}^{2}} \mathrm{~d} \lambda_{n} \\
& =-\sum_{i=1}^{n} \int_{-r}^{r} \cdots \int_{-r}^{r} \frac{\partial \omega_{k}}{\partial x_{i}}(\boldsymbol{x}) \frac{\partial h}{\partial x_{i}}(\boldsymbol{x}) \mathrm{d} x_{i} \mathrm{~d} x_{1} \cdots \mathrm{d} x_{i-1} \mathrm{~d} x_{i+1} \cdots \mathrm{d} x_{n} \\
& =-\sum_{i=1}^{n} \int_{-r}^{r} \cdots \int_{-r}^{r}\left\{\left(\int_{-r}^{-M_{i}}+\int_{M_{i}}^{r}\right) \operatorname{sign} x_{i} \omega_{k-1}(\boldsymbol{x}) \frac{\partial h}{\partial x_{i}}(\boldsymbol{x}) \mathrm{d} x_{i}\right\} \mathrm{d} x_{1} \cdots \mathrm{d} x_{i-1} \mathrm{~d} x_{i+1} \cdots \mathrm{d} x_{n} \\
& =-\sum_{i=1}^{n} \int_{-r}^{r} \cdots \int_{-r}^{r}\left\{\int_{M_{i}}^{r} \omega_{k-1}(\boldsymbol{x}) \frac{\partial}{\partial x_{i}}\left[h\left(\boldsymbol{x}_{-x_{i}}^{i}\right)+h(\boldsymbol{x})\right] \mathrm{d} x_{i}\right\} \mathrm{d} x_{1} \cdots \mathrm{d} x_{i-1} \mathrm{~d} x_{i+1} \cdots \mathrm{d} x_{n} .
\end{aligned}
$$


Hence, we have

$$
0=-\sum_{i=1}^{n} \int_{-r}^{r} \cdots \int_{-r}^{r}\left\{h\left(x_{-r}^{i}\right)+h\left(x_{+r}^{i}\right)-\left[h\left(x_{-M_{i}}^{i}\right)+h\left(x_{+M_{i}}^{i}\right)\right]\right\} \mathrm{d} x_{1} \cdots \mathrm{d} x_{i-1} \mathrm{~d} x_{i+1} \cdots \mathrm{d} x_{n}
$$

if $k=1$ and

$$
\begin{aligned}
0= & -\sum_{i=1}^{n} \int_{-r}^{r} \cdots \int_{-r}^{r} \int_{M_{i}}^{r} \omega_{k-2}(\boldsymbol{x})\left[h\left(\boldsymbol{x}_{-x_{i}}^{i}\right)+h(\boldsymbol{x})\right] \mathrm{d} x_{i} \mathrm{~d} x_{1} \cdots \mathrm{d} x_{i-1} \mathrm{~d} x_{i+1} \cdots \mathrm{d} x_{n} \\
& +\sum_{i=1}^{n} \int_{-r}^{r} \cdots \int_{-r}^{r} \omega_{k-1}\left(\boldsymbol{x}_{+M_{i}}^{i}\right)\left[h\left(\boldsymbol{x}_{-M_{i}}^{i}\right)+h\left(\boldsymbol{x}_{+M_{i}}^{i}\right)\right] \mathrm{d} x_{1} \cdots \mathrm{d} x_{i-1} \mathrm{~d} x_{i+1} \cdots \mathrm{d} x_{n}
\end{aligned}
$$

if $k \geq 2$.

Clearly, (5) is equivalent to (3) and from (6) it follows

$$
0=\int_{I_{n}} \Delta h \mathrm{~d} \lambda_{n}^{\omega_{k}}=\int_{I_{n}} h \mathrm{~d} \lambda_{n}^{\omega_{k-2}}-2 \int_{D_{n}} h \mathrm{~d} \lambda_{n-1}^{\omega_{k-1}},
$$

which is equivalent to (4).

Let $\quad M:=M(\boldsymbol{x}):=\max _{1 \leq i \leq n}\left|x_{i}\right|$. Analogously to the proof of Theorem 1 (ii), Equation (7) is generalized to:

Corollary 1 If $h \in \mathcal{H}\left(I_{n}\right)$ and $\varphi \in C^{2}[0, r]$ is such that $\varphi(0)=0$ and $\varphi^{\prime}(0)=0$, then

$$
0=\int_{I_{n}} \varphi(r-M) \Delta h \mathrm{~d} \lambda_{n}=\int_{I_{n}} \varphi^{\prime \prime}(r-M) h \mathrm{~d} \lambda_{n}-2 \int_{D_{n}} \varphi^{\prime}(r-M) h \mathrm{~d} \lambda_{n-1} .
$$

The volume mean-value formula (2) was extended by P. Pizzetti to the following [2] [3] [8].

The Pizzetti formula. If $g \in \mathcal{H}^{m}\left(B_{n}(r)\right)$, then

$$
\int_{B_{n}(r)} g \mathrm{~d} \lambda_{n}=r^{n} \pi^{n / 2} \sum_{k=0}^{m-1} \frac{r^{2 k}}{2^{2 k} \Gamma(n / 2+k+1)} \frac{\Delta^{k} g(\mathbf{0})}{k !} .
$$

Here, we present a similar formula for polyharmonic functions on the hypercube based on integration over the set $D_{n}$.

Theorem 2 If $g \in \mathcal{H}^{m}\left(I_{n}\right), m \geq 1$, and $\varphi \in C^{2 m}[0, r]$ is such that $\varphi^{(k)}(0)=0, k=0,1, \cdots, 2 m-1$, then the following identity holds true for any $k \geq 0$ :

$$
\int_{I_{n}} \varphi^{(2 m)}(r-M) g \mathrm{~d} \lambda_{n}=2 \sum_{s=0}^{m-1} \int_{D_{n}} \varphi^{(2 s+1)}(r-M) \Delta^{m-s-1} g \mathrm{~d} \lambda_{n-1},
$$

where $\varphi^{(j)}(t)=\frac{\mathrm{d}^{j} \varphi}{\mathrm{d} t^{j}}(t)$.

Proof. Equation (9) is a direct consequence from (8):

$$
\begin{aligned}
0 & =\int_{I_{n}} \varphi(r-M) \Delta^{m} g \mathrm{~d} \lambda_{n} \\
& =-2 \int_{D^{n}} \varphi^{(1)}(r-M) \Delta^{m-1} g \mathrm{~d} \lambda_{n-1}+\int_{I_{n}} \varphi^{(2)}(r-M) \Delta^{m-1} g \mathrm{~d} \lambda_{n} \\
& =\cdots=-2 \sum_{s=0}^{m-1} \int_{D_{n}} \varphi^{(2 s+1)} \Delta^{m-s-1} g \mathrm{~d} \lambda_{n-1}+\int_{I_{n}} \varphi^{(2 m)} g \mathrm{~d} \lambda_{n} .
\end{aligned}
$$

\section{A Relation to Best One-Sided $L^{1-A p p r o x i m a t i o n ~ b y ~ H a r m o n i c ~ F u n c t i o n s ~}$}

Theorem 1 suggests that for a certain positive cone in $C\left(I_{n}\right)$ the set $D_{n}$ is a characteristic set for the best one-sided $L^{1}$-approximation with respect to $\mathcal{H}\left(I_{n}\right)$ as it is explained and illustrated by the examples presented below.

For a given $f \in C\left(I_{n}\right)$, let us introduce the following subset of $\mathcal{H}\left(I_{n}\right)$ :

$$
\mathcal{H}_{-}\left(I_{n}, f\right):=\left\{h \in \mathcal{H}\left(I_{n}\right) \mid h \leq f \text { on } I_{n}\right\} .
$$

A harmonic function $h_{*}^{f} \in \mathcal{H}_{-}\left(I_{n}, f\right)$ is said to be a best one-sided $L^{1}$-approximant from below to $f$ with respect to $\mathcal{H}\left(I_{n}\right)$ if 


$$
\left\|f-h_{*}^{f}\right\|_{1} \leq\|f-h\|_{1} \text { for every } h \in \mathcal{H}_{-}\left(I_{n}, f\right),
$$

where

$$
\|g\|_{1}:=\int_{I_{n}}|g| \mathrm{d} \lambda_{n} .
$$

Theorem 1 (ii) readily implies the following ([6] [9]).

Theorem 3 Let $f \in C\left(I_{n}\right)$ and $h_{*}^{f} \in \mathcal{H}_{-}\left(I_{n}, f\right)$. Assume further that the set $D_{n}$ belongs to the zero set of the function $f-h_{*}^{f}$. Then $h_{*}^{f}$ is a best one-sided $L^{1}$-approximant from below to $f$ with respect to $\mathcal{H}\left(I_{n}\right)$.

Corollary 2 If $f \in C^{1}\left(I_{n}\right)$, any solution $h$ of the problem

$$
h_{\mid D_{n}}=f_{\mid D_{n}}, \nabla h_{\mid D_{n}}=\nabla f_{\mid D_{n}}, h \in \mathcal{H}_{-}\left(I_{n}, f\right),
$$

is a best one-sided $L^{1}$-approximant from below to $f$ with respect to $\mathcal{H}\left(I_{n}\right)$.

Corollary 3 If $f(x)=g(x) \prod_{1 \leq i<j \leq n}\left(x_{i}^{2}-x_{j}^{2}\right)^{2}$, where $g \in C\left(I_{n}\right)$ and $g \geq 0$ on $I_{n}$, then $h_{*}^{f}(x) \equiv 0$ is a best one-sided $L^{1}$-approximant from below to $f$ with respect to $\mathcal{H}\left(I_{n}\right)$.

Example 1 Let $n=2, r=1$ and $f_{1}\left(x_{1}, x_{2}\right)=x_{1}^{2} x_{2}^{2}$. By Corollary 2, the solution $h_{*}^{f_{1}}\left(x_{1}, x_{2}\right)=-x_{1}^{4} / 4+\frac{3}{2} x_{1}^{2} x_{2}^{2}-x_{2}^{4} / 4$ of the interpolation problem (10) with $f=f_{1}$ is a best one-sided $L^{1}$ appro-ximant from below to $f_{1}$ with respect to $\mathcal{H}\left(I_{2}\right)$ and $\left\|f_{1}-h_{*}^{f_{1}}\right\|_{1}=8 / 45$. Since the function $f_{1}$ belongs to the positive cone of the partial differential operator $\mathcal{D}_{2,2}^{4}:=\frac{\partial^{4}}{\partial x_{1}^{2} \partial x_{2}^{2}}$ (that is, $\mathcal{D}_{2,2}^{4} f_{1}>0$ ), one can compare the best harmonic one-sided $L^{1}$-approximation to $f_{1}$ with the corresponding approximation from the linear subspace of $C\left(I_{2}\right)$ :

$$
\mathcal{B}^{2,2}\left(I_{2}\right):=\left\{b \in C\left(I_{2}\right) \mid b\left(x_{1}, x_{2}\right)=\sum_{j=0}^{1}\left[a_{0 j}\left(x_{1}\right) x_{2}^{j}+a_{1 j}\left(x_{2}\right) x_{1}^{j}\right]\right\} .
$$

The possibility for explicit constructions of best one-sided $L^{1}$-approximants from $\mathcal{B}^{2,2}\left(I_{2}\right)$, is studied in [10]. The functions $f_{1}-b_{*}^{f_{1}}$ and $f_{1}-b_{f_{1}}^{*}$, where $b_{*}^{f_{1}}$ and $b_{f_{1}}^{*}$ are the unique best one-sided $L^{1}$-approximants to $f_{1}$ with respect to $\mathcal{B}^{2,2}\left(I_{2}\right)$ from below and above, respectively, play the role of basic error functions of the canonical one-sided $L^{1}$-approximation by elements of $\mathcal{B}^{2,2}\left(I_{2}\right)$. For instance, $b_{*}^{f_{1}}$ can be constructed as the unique interpolant to $f_{1}$ on the boundary $\diamond:=\left\{\left(x_{1}, x_{2}\right) \in I_{2}|| x_{1}|+| x_{2} \mid=1\right\}$ of the inscribed square and $\left\|f_{1}-b_{*}^{f_{1}}\right\|_{1}=14 / 45$ (Figure 2).

Example 2 Let $n=2, r=1$ and $f_{2}\left(x_{1}, x_{2}\right)=x_{1}^{8}+14 x_{1}^{4} x_{2}^{4}+x_{2}^{8}$. The solution $h_{*}^{f_{2}}\left(x_{1}, x_{2}\right)=x_{1}^{8}+x_{2}^{8}-28\left(x_{1}^{6} x_{2}^{2}+x_{1}^{2} x_{2}^{6}\right)+70 x_{1}^{4} x_{2}^{4}$ of (10) with $f=f_{2}$ is a best one-sided $L^{1}$-approximant from below to $f_{2}$ with respect to $\mathcal{H}\left(I_{2}\right)$ and $\left\|f_{2}-h_{*}^{f_{2}}\right\|=8 / 75$. It can also be verified that $\left\|f_{2}-b_{*}^{f_{2}}\right\|=121 / 900$ (see Figure 3).
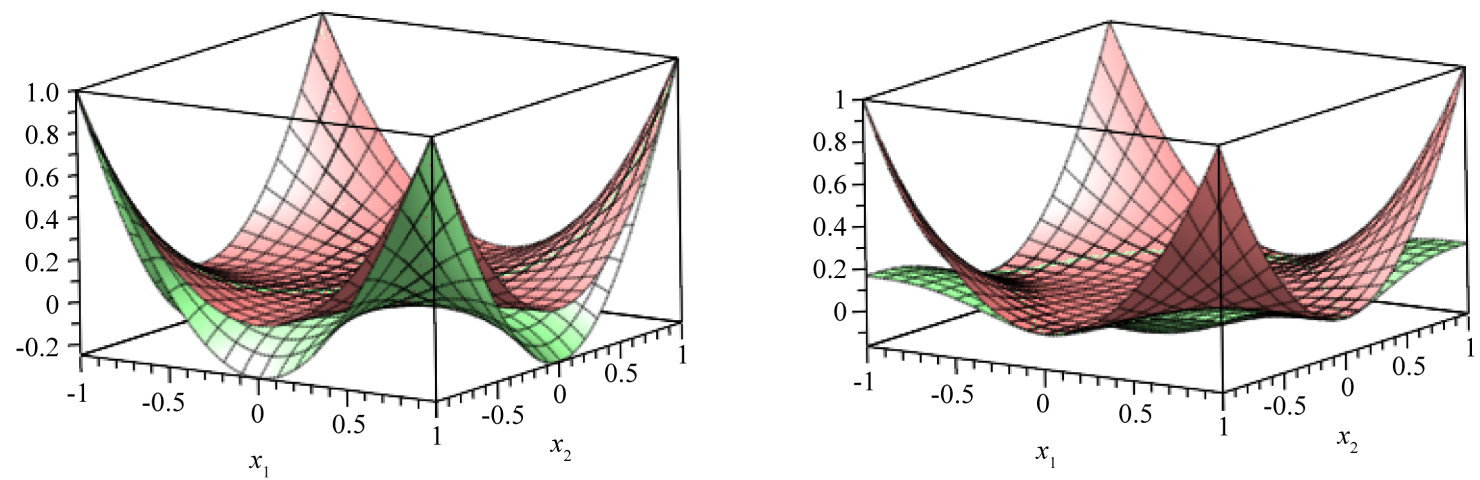

Figure 2. The graphs of the function $f_{1}\left(x_{1}, x_{2}\right)=x_{1}^{2} x_{2}^{2}$ (coral) and its best one-sided $L^{1}$-approximants from below, $h_{*}^{f_{1}}$ with respect to $H\left(I_{2}\right)$ (left) and $b_{*}^{f_{1}}$ with respect to $\mathcal{B}^{2,2}\left(I_{2}\right)$ (right). 

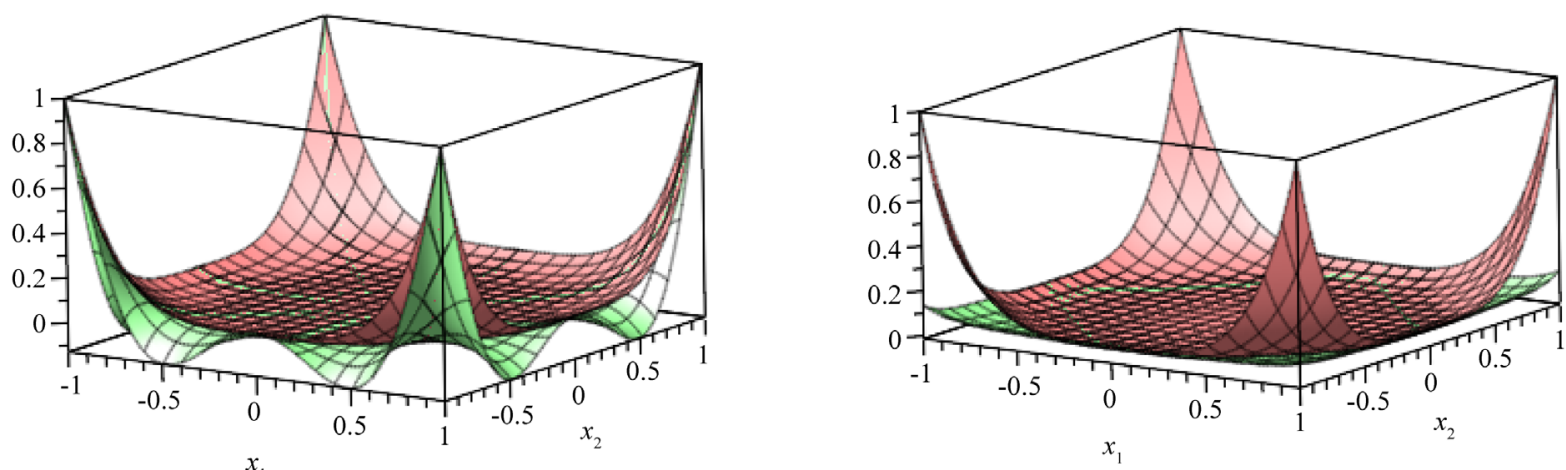

Figure 3. The graphs of the function $f_{2}\left(x_{1}, x_{2}\right)=x_{1}^{8}+14 x_{1}^{4} x_{2}^{4}+x_{2}^{8}$ (coral) and its best one-sided $L^{1}$-approximants from below, $h_{*}^{f_{2}}$ with respect to $\mathcal{H}\left(I_{2}\right)$ (left) and $b_{*}^{f_{2}}$ with respect to $\mathcal{B}^{2,2}\left(I_{2}\right)$ (right).

Remark 1 Let $\varphi \in C^{2}[0, r]$ is such that $\varphi(0)=0, \varphi^{\prime}(0)=0$, and $\varphi^{\prime} \geq 0, \varphi^{\prime \prime} \geq 0$ on $[0, r]$. It follows from (8) that Theorem 3 also holds for the best weighted $L^{1}$-approximation from below with respect to $\mathcal{H}\left(I_{n}\right)$ with weight $\varphi^{\prime \prime}(r-M)$. The smoothness requirements were used for brevity and wherever possible they can be weakened in a natural way.

\section{References}

[1] Helms, L.-L. (2009) Potential Theory. Springer-Verlag, London. http://dx.doi.org/10.1007/978-1-84882-319-8

[2] Pizzetti, P. (1909) Sulla media dei valori che una funzione dei punti dello spazio assume sulla superficie della sfera. Rendiconti Linzei-Matematica e Applicazioni, 18, 182-185.

[3] Courant, R. and Hilbert, D. (1989) Methods of Mathematical Physics Vol. II. Partial Differential Equations Reprint of the 1962 Original. John Wiley \& Sons Inc., New York.

[4] Goldstein, M., Haussmann, W. and Rogge, L. (1988) On the Mean Value Property of Harmonic Functions and Best Harmonic $L^{1}$-Approximation. Transactions of the American Mathematical Society, 305, 505-515.

[5] Sakai, M. (1982) Quadrature Domains. Lecture Notes in Mathematics, Springer, Berlin.

[6] Gustafsson, B., Sakai, M. and Shapiro, H.S. (1977) On Domains in Which Harmonic Functions Satisfy Generalized Mean Value Properties. Potential Analysis, 71, 467-484.

[7] Gustafsson, B. (1998) On Mother Bodies of Convex Polyhedra. SIAM Journal on Mathematical Analysis, 29, 11061117. http://dx.doi.org/10.1137/S0036141097317918

[8] Bojanov, B. (2001) An Extension of the Pizzetti Formula for Polyharmonic Functions. Acta Mathematica Hungarica, 91, 99-113. http://dx.doi.org/10.1023/A:1010687011674

[9] Armitage, D.H. and Gardiner, S.J. (1999) Best One-Sided $L^{1}$-Approximation by Harmonic and Subharmonic Functions. In: Haußmann, W., Jetter, K. and Reimer, M., Eds., Advances in Multivariate Approximation, Mathematical Research (Volume 107), Wiley-VCH, Berlin, 43-56.

[10] Dryanov, D. and Petrov, P. (2002) Best One-Sided $L^{1}$-Approximation by Blending Functions of Order $(2,2)$. Journal of Approximation Theory, 115, 72-99. http://dx.doi.org/10.1006/jath.2001.3652 